\title{
Prospective feasibility study of sealing pulmonary vessels with energy in lung surgery
}

\author{
Morihito Okada, MD, PhD, ${ }^{a}$ Yoshihiro Miyata, MD, PhD, ${ }^{a}$ Kazuya Takamochi, MD, PhD, ${ }^{b}$ \\ Yasuhiro Tsutani, MD, PhD, ${ }^{\mathrm{a}}$ Shiaki Oh, MD, PhD, ${ }^{\mathrm{b}}$ and Kenji Suzuki, MD, $\mathrm{PhD}^{\mathrm{b}}$
}

\section{ABSTRACT}

Objective: Vascular sealing with an energy vessel sealing system during lung resection may allow surgeons to treat small vessels with minimal dissection, possibly decreasing likelihood of injury. Few large prospective trials have examined the proximal sides of vessels not ligated in addition to sealing during surgery. We therefore assessed feasibility of an energy device to seal pulmonary artery and vein branches without further ligation.

Methods: This prospective, preoperative registration study at 2 institutions evaluated safety of energy sealing with the LigaSure (Medtronic, Minneapolis, Minn), with no additional reinforcing material such as suture ligation, for pulmonary vessels as large as $7 \mathrm{~mm}$ during anatomic lung resection (cohort 1 study). A postoperative hemorrhage occurred in the 128th case, so a cohort 2 study proceeded after we changed inclusion criterion for pulmonary arteries from a maximum of $7 \mathrm{~mm}$ to a maximum of $5 \mathrm{~mm}$.

Results: In cohort $1(n=128)$ and cohort $2(n=200), 216$ and 250 pulmonary arteries and 189 and 213 pulmonary veins, respectively, were treated with energy sealing. Overall postoperative hemorrhage rate was $0.3 \%$ (1/328 patients); however, no serious postoperative complications were associated with energy sealing among the 200 patients in cohort 2 . Subsequent inspection of the torn artery stump confirmed that the bleeding in the 128th case was in an area adjacent to the sealing zone.

Conclusions: Energy sealing without reinforcement allows secure treatment during lung resection of pulmonary arteries as large as $5 \mathrm{~mm}$ in diameter and pulmonary veins as large as 7 mm. (J Thorac Cardiovasc Surg 2019;157:388-95)

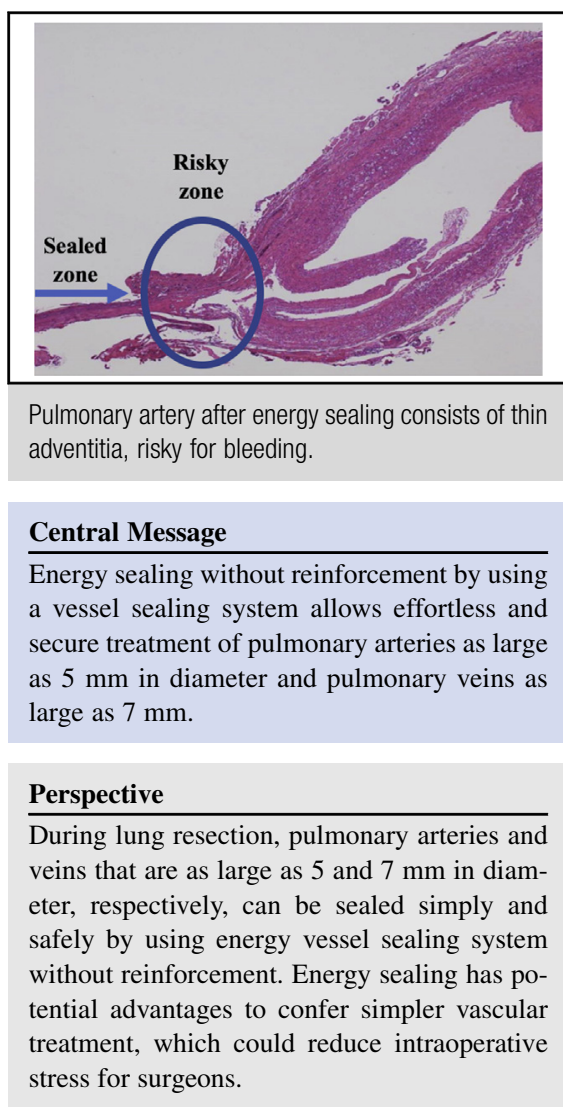

See Editorial Commentary page 396.

See Editorial page 378 .

\footnotetext{
From the a Department of Surgical Oncology, Hiroshima University, Hiroshima, Japan; and ${ }^{\mathrm{b}}$ Department of Thoracic Surgery, Juntendo University, Japan.

This study was not supported by any funding.

Read at the 98th Annual Meeting of The American Association for Thoracic Surgery, San Diego, California, April 28-May 1, 2018.

Received for publication Aug 23, 2017; revisions received Jan 14, 2018; accepted for publication Feb 11, 2018; available ahead of print Nov 8, 2018.

Address for reprints: Morihito Okada, MD, PhD, Department of Surgical Oncology, Research Institute for Radiation Biology and Medicine Hiroshima University, 1-2-

3-Kasumi, Minami-ku, Hiroshima City, Hiroshima 734-0037, Japan (E-mail: morihito@hiroshima-u.ac.jp).

$0022-5223 / \$ 36.00$

Copyright (c) 2018 by The American Association for Thoracic Surgery

https://doi.org/10.1016/j.jtcvs.2018.02.113
}

Anatomic lung resection for pulmonary pathologies such as lung cancer includes lobectomy and segmentectomy. Advances in radiographic equipment and the widespread application of low-dose helical computed tomography for

Scanning this QR code will take you to a supplemental video. To view the AATS Annual Meeting Webcast, see the URL next to the webcast thumbnail.

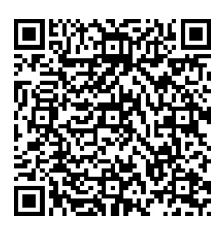




\section{Abbreviation and Acronym \\ VATS $=$ video-assisted thoracoscopic surgery}

screening have increased the early detection of small tumors, particularly adenocarcinoma, and segmentectomy has therefore become a more popular procedure. ${ }^{1,2}$ Accordingly, surgeons now have more opportunities to access small vessels during lung cancer surgery. Videoassisted thoracoscopic surgery (VATS) has become the preferred surgical approach for treating many types of pulmonary diseases, and simple treatment of tiny pulmonary vessels is important for surgeons because suture ligation through thoracoscopic ports is challenging. Because of these trends, many surgeons would welcome simple and safe methods of ligating and sealing vascular branches.

Sealing small vessels with energy devices might be superior to ligation by hand or management with staplers. Energy sealing could remove the need for excessive tissue dissection around vessels, which could consequently reduce the incidence of intraoperative injuries. ${ }^{3-5}$ The procedures should be even simpler for preventing the postoperative complications, especially after critical lung surgery. ${ }^{6,7}$ To our knowledge, the safety and effectiveness of energy sealing for pulmonary vessels has not been addressed in prospective, multi-institution studies. The primary objective of this study was to determine the feasibility of sealing small vascular vessels with energy devices during anatomic lung resection.

\section{PATIENTS AND METHODS \\ Cohort 1}

We started a prospective study at 2 institutions (Hiroshima University, Hiroshima, Japan, and Juntendo University, Tokyo, Japan) from January 2013 to evaluate the safety of energy sealing for small vessels during anatomic lung resection consisting of lobectomy and segmentectomy. The primary end point was the frequency of hemorrhage within 28 days after surgery. The inclusion criteria were as follows: age at least 20 years, operable cancer with planned anatomic lung resection, sufficient function of major organs, and an Eastern Cooperative Oncology Group performance status score of 0 or 1 . Patients had to be capable of comprehending the full nature and purpose of the study, as well as possible risks and side effects. They also had to cooperate with the investigator, understand oral or written instructions, and comply with the requirements of the entire study. Patients voluntarily signed an informed consent form approved by the institutional review board and independent ethics committee at the institution where they participated before starting any procedures that were specific to the study. The trial registration number is UMIN000018493. The exclusion criteria included having undergone induction neoadjuvant chemoradiotherapy or long-term treatment with oral steroids, which can increase the vulnerability of the tissue, and having a history or evidence of any other clinically significant disorder, condition, or disease that in the opinion of the investigator would pose a risk to patient safety or interfere with the study evaluation, procedures, or completion. It is clearly described in the protocol that the trial must be stopped as soon as possible if it is surgically confirmed that a patient has had a bleeding event develop in the manipulated blood vessel within 28 days after the thoracotomy. The target enrollment was 200 patients
(100 per institute). Ueda and colleagues ${ }^{8}$ reported that the incidence of bleeding exceeding $500 \mathrm{~mL}$ was around $1.5 \%$ after thoracic and cardiovascular surgery in Japan during 2007. Sample size was calculated using binomial distribution. Under the alternative hypothesis rate of $1.5 \%$, 200 patients were required to observe 1 or more events with a probability of $95 \%$ or more. The energy sealing device was LigaSure Blunt Tip (advanced bipolar type, straight tip, $5 \mathrm{~mm}$ in width; Medtronic, Minneapolis, Minn), a handheld electrothermal device that can seal vessels as large as $7 \mathrm{~mm}$ in diameter. Patients eligible for lobectomy or segmentectomy were registered before undergoing surgery. Patients were treated with either VATS or muscle-sparing thoracotomy in accordance with accepted clinical practice standards. Pulmonary arteries and veins as large as $7 \mathrm{~mm}$ were sealed with the LigaSure device, and blood vessels larger than $7 \mathrm{~mm}$ were closed with an endostapler. The branches of pulmonary vessels were isolated, and a shortened sterile paper ruler was inserted inside the chest to measure their diameters at the level where they would be cut. The isolated vessel branches were then divided with a single application of the LigaSure instrument, without additional applications or procedures such as suture ligation or clips. The bronchi and the lung parenchyma were treated with conventional electrocautery and endostaplers, but no other special equipment was used (Video 1). Both institutional ethical committees approved this study, the purpose of which was explained in detail to all patients, who then provided written, informed consent before undergoing operation. Because postoperative bleeding occurred in the 128th case in this series, the protocol was stopped, and the study was immediately abandoned.

\section{Cohort 2}

We changed 1 inclusion criterion and reduced the maximum size of the pulmonary artery to $5 \mathrm{~mm}$. The maximum size of the pulmonary vein remained at $7 \mathrm{~mm}$, and the cohort 2 study was started in October 2015 . By April 2017, a total of 200 patients were enrolled (100 per institution), and no severe complications, such as postoperative bleeding occurred. In cohort 2, the LigaSure Maryland Jaw (advanced bipolar type, straight tip, $5 \mathrm{~mm}$ in width; Medtronic) was used.

\section{RESULTS}

Figure 1 shows the participant flow chart. During the cohort 1 study, there were 216 and 189 pulmonary arteries and veins, respectively, that were energy sealed in 128 patients, and 250 and 213 pulmonary arteries and veins, respectively, that were energy sealed in the cohort 2 study of 200 patients. We analyzed results from a total of 328 patients, even though 539 patients provided preoperative, informed consent to participate in this study, because the vessels in 211 patients, who had no small vessels meeting the required conditions of the protocol, were not energy sealed. After each blood vessel was assessed intraoperatively for size with a sterile paper ruler, the branch pulmonary artery and pulmonary veins to be divided were larger than 5 and $7 \mathrm{~mm}$ respectively, in 211 patients and therefore treated with staples. There were no cases in which the vessel size was suitable but the surgeon decided not to seal with the LigaSure device. There were no differences in the baseline characteristics between the 328 patients who underwent vessel sealing with energy device and the 211 patients who did not.

Table 1 shows that the characteristics did not significantly differ between the patients in cohort $1(n=128)$ and cohort $2(\mathrm{n}=200)$. The total patients $(\mathrm{n}=328)$ were 184 men $(56 \%)$ and 144 women $(44 \%)$ aged 27 to 90 years (median, 


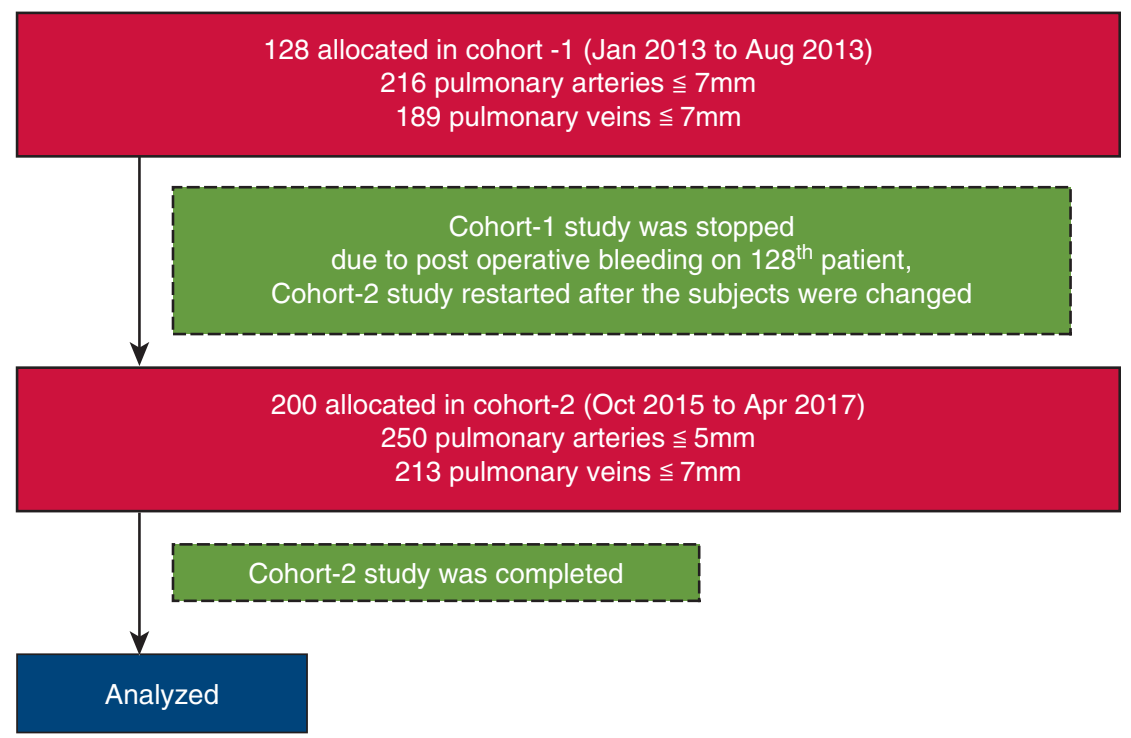

FIGURE 1. Participant flowchart.

68 years). Surgery was required to treat lung cancer and metastatic tumors in $307(94 \%)$ and $8(6 \%)$ patients, respectively. The types of anatomic resection were lobectomy and segmentectomy in $240(73 \%)$ and 88 patients $(27 \%)$, respectively. Comorbidities comprised emphysema, diabetes, and ischemic heart disease in $53(16 \%), 33(10 \%)$, and $29(9 \%)$ patients, respectively.

Figure 2 shows the sizes of vessels sealed with the LigaSure. According to protocol, none of the treated pulmonary arteries in cohort 2 were larger than $5 \mathrm{~mm}$ in diameter. Table 2 shows the surgical outcomes. Only 1 patient $(0.3 \%)$ had postoperative hemorrhage, which was the reason for stopping the initial cohort 1 study. Hemorrhage did not arise in cohort 2. Two of 328 patients $(0.6 \%)$ required blood transfusions, and the median amount of blood lost during procedures was $26 \mathrm{~mL}$ (range, 0-2007 $\mathrm{mL}$ ). The median surgical duration was 136 minutes (range, 51-447 minutes). At 30 days after the surgery, there were neither in-hospital deaths nor operative deaths.

The pulmonary artery around the energy sealing site was histopathologically examined without incident. The results indicated that the sealed zone was complete, but the adjacent portion exclusively comprised a very thin layer of adventitia, because the intima and media were replaced after

TABLE 1. Patient characteristics

\begin{tabular}{|c|c|c|c|c|}
\hline & Cohort $1(n=128)$ & Cohort $2(n=200)$ & Total $(\mathbf{n}=\mathbf{3 2 8})$ & $P$ value \\
\hline Age (y, median and IQR) & $66(59-72)$ & $70(63-76)$ & $68(61-74)$ & .001 \\
\hline $\begin{array}{l}\text { Sex } \\
\qquad \text { Male } \\
\text { Female }\end{array}$ & $\begin{array}{l}71(55 \%) \\
57(45 \%)\end{array}$ & $\begin{array}{r}113(56 \%) \\
87(44 \%)\end{array}$ & $\begin{array}{l}184(56 \%) \\
144(44 \%)\end{array}$ & .854 \\
\hline $\begin{array}{l}\text { Pathology } \\
\text { Lung cancer } \\
\text { Metastasis } \\
\text { Others }\end{array}$ & $\begin{array}{c}115(90 \%) \\
4(3 \%) \\
9(7 \%)\end{array}$ & $\begin{array}{c}192(96 \%) \\
4(2 \%) \\
4(2 \%)\end{array}$ & $\begin{array}{c}307(94 \%) \\
8(2.4 \%) \\
13(4 \%)\end{array}$ & .238 \\
\hline $\begin{array}{l}\text { Procedure } \\
\text { Lobectomy } \\
\text { Segmentectomy }\end{array}$ & $\begin{array}{l}95(74 \%) \\
33(26 \%)\end{array}$ & $\begin{array}{r}145(73 \%) \\
55(27 \%)\end{array}$ & $\begin{array}{r}240(73 \%) \\
88(27 \%)\end{array}$ & .732 \\
\hline \multicolumn{5}{|l|}{ Comorbidity } \\
\hline Diabetes & $11(9 \%)$ & $22(11 \%)$ & $33(10 \%)$ & .574 \\
\hline Ischemic heart disease & $9(7 \%)$ & $20(10 \%)$ & $29(9 \%)$ & .428 \\
\hline Brain infarction & $2(2 \%)$ & $5(3 \%)$ & $7(2 \%)$ & .709 \\
\hline Anticoagulation drug & $5(4 \%)$ & $13(7 \%)$ & $18(5 \%)$ & .457 \\
\hline Emphysema & $12(9 \%)$ & $41(21 \%)$ & $53(16 \%)$ & .009 \\
\hline Pulmonary fibrosis & $5(4 \%)$ & $10(5 \%)$ & $15(5 \%)$ & .789 \\
\hline
\end{tabular}

Data are $\mathrm{n}(\%)$ unless marked otherwise. $I Q R$, Interquartile range. 


\section{Size of treated vessels}
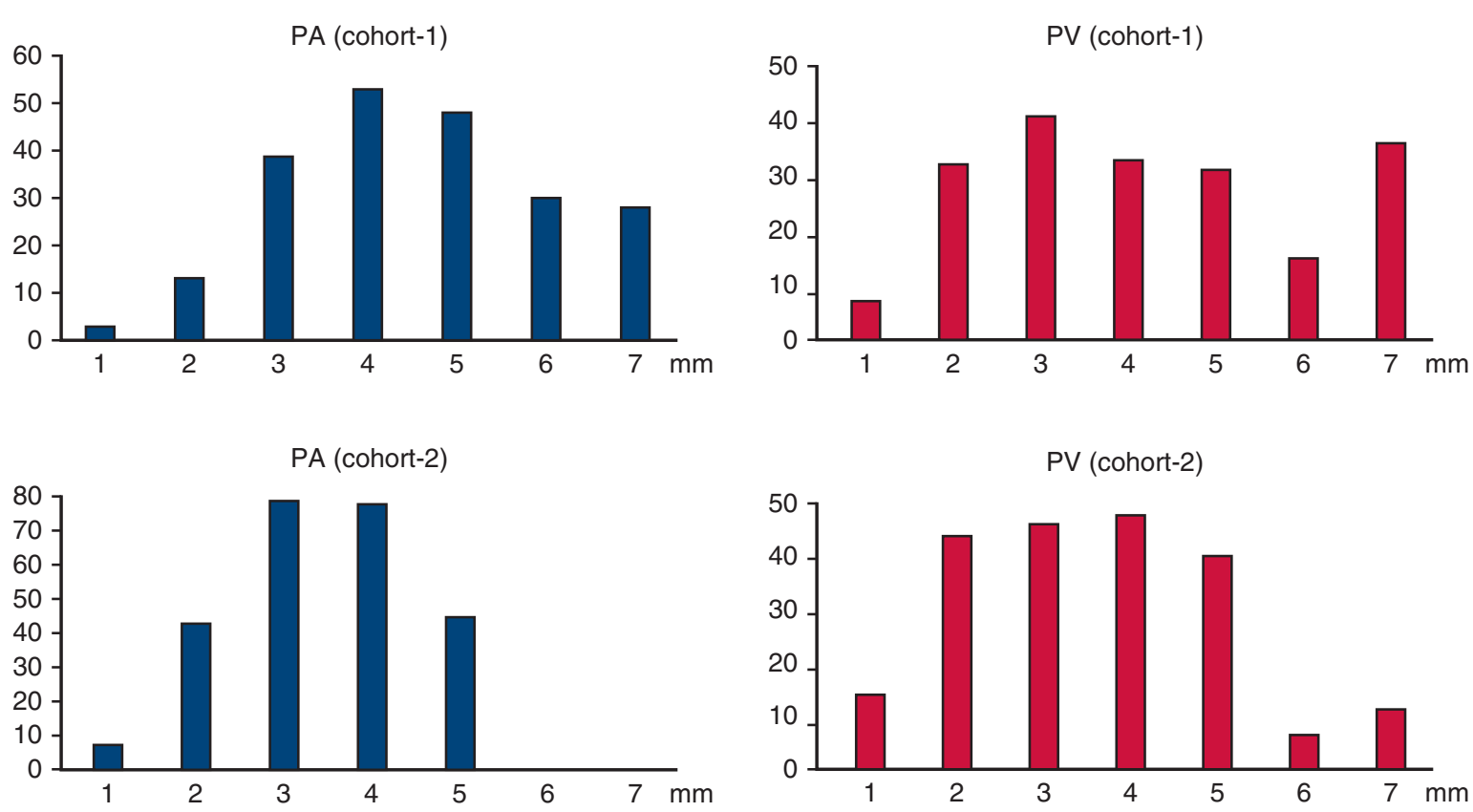

FIGURE 2. Sizes of treated pulmonary artery $(P A)$ and pulmonary vein $(P V)$ in cohort 1 (top) and cohort 2 (bottom).

invagination into the vessel lumen (Figure 3,A). This thin layer was susceptible to a high risk of bleeding, and we speculate that the postoperative bleeding occurred at such a site in the 128th patient. Vulnerable areas adjacent to sealed zones were not identified in pulmonary veins (Figure 3, $B$ ).

\section{Patient With Postoperative Bleeding}

Patient 128, a 63-year-old man with high blood pressure and a history of 2 subarachnoid hemorrhages had been prescribed antihypertensive drugs. He underwent upper lobectomy of the left lung without complications. The $\mathrm{A} 1+2 \mathrm{~b}$ (dorsalis of apicodorsalis) and $\mathrm{A} 1+2 \mathrm{c}$ (horizontalis of apicodorsalis) branches of the pulmonary artery were sealed with the LigaSure Blunt Tip. A postprocedural chest radiograph taken after a postural change to the supine position did not reveal any bleeding. During recovery from anesthesia, the patient violently moved by himself, and his blood pressure increased to around $200 \mathrm{~mm} \mathrm{Hg}$. Immediately after an anesthesiologist injected him with an antihypertensive drug and removed the endotracheal intubation tube, the amount of bleeding from the chest drainage tube suddenly increased.

The patient was placed under anesthesia and reintubated, and another thoracotomy was prepared. Bleeding around the stump of the $\mathrm{A} 1+2 \mathrm{~b}$ (diameter, $6 \mathrm{~mm}$ ) branch that had been sealed with the LigaSure was quickly stopped by application of light compression with gauze. The more proximal side of the artery was ligated, and the stumps were removed for investigation. A fissure surrounding the intact tip portion sealed with the LigaSure was considered as the source of the bleeding (Figure 4). Postoperative video inspection

TABLE 2. Surgical outcomes

\begin{tabular}{|c|c|c|c|c|}
\hline & Cohort $1(n=128)$ & Cohort $2(n=200)$ & Total $(\mathbf{n}=328)$ & $P$ value \\
\hline No. of treated pulmonary arteries & 216 & 250 & 466 & .001 \\
\hline No. of treated pulmonary veins & 189 & 213 & 402 & .016 \\
\hline Blood loss (mL, median and IQR) & $30(17-50)$ & $25(10-50)$ & $26(13-50)$ & .220 \\
\hline Operative duration (min, median and IQR) & $143(20-155)$ & $132(104-164)$ & $136(86-161)$ & .619 \\
\hline Blood transfusion & $1(0.8 \%)$ & $1(0.5 \%)$ & $2(0.6 \%)$ & $>.999$ \\
\hline Postoperative bleeding & & & & .390 \\
\hline Pulmonary artery & $1(0.8 \%)$ & 0 & $1(0.3 \%)$ & \\
\hline Pulmonary vein & 0 & 0 & 0 & \\
\hline
\end{tabular}

Data are $\mathrm{n}(\%)$ unless marked otherwise. IQR, Interquartile range. 

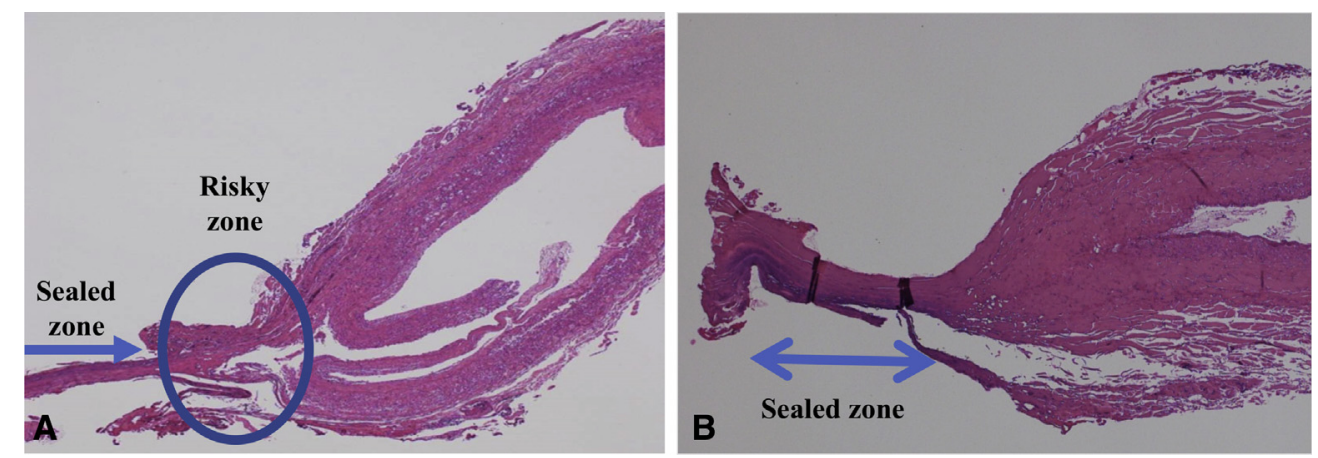

FIGURE 3. Representative histopathologic findings of pulmonary artery (A) and vein (B) divided with an energy device. The pulmonary artery is completely sealed; however, the adjacent portion consists exclusively of a very thin layer adventitia, because intima and media were replaced after invagination into vessel lumen. This area adjacent to sealed portion is susceptible to bleeding. No such area is evident in the pulmonary vein.

confirmed that the status of the sealed portions of the vessel stumps was acceptable. The patient recovered uneventfully.

\section{DISCUSSION}

Handheld energy devices might present a potential modality for sealing and cutting branches of the pulmonary vessels, which would reduce the need for vascular manipulation and dissection over a wide area. They might also improve accessibility to small vessels located in areas that are difficult to reach with suture ligation and vascular staplers. To date, except for 1 study, ${ }^{3}$ no large, prospective human trials have investigated the proximal side of vessels that were not ligated in addition to sealing during surgery. The primary goal of this study was to determine the immediate safety of applying energy sealing to pulmonary arteries and veins during anatomic lung resection. We assessed short-term postoperative hemorrhagic complications of sealed vessel stumps that are associated with fatal morbidity and mortality. Whether energy devices would reduce the surgical duration was dependent on whether energy sealing can be used with or without proximally placed ligation. This remains a significant topic, because many surgeons believe that suture ligation or clips placed on the proximal side are needed to ensure safety. The safety of energy devices with further ligations on the proximal sides of vessels has been described. ${ }^{9}$ A single application of an energy device without additional ligation, 1-step direct sealing, is clinically important for treating vessel branches. The manipulation and division of small pulmonary vessels, especially arteries, during anatomic lung resection is potentially hazardous, especially through the VATS approach. Vascular staplers, however, remain the main instrument for dividing and cutting pulmonary vessels during VATS lobectomy. Energy sealing devices require less manipulation and dissection and are thus easier to apply than staplers. The LigaSure device has been widely used, and it has been considered safe for the universal sealing of vessels as large as $7 \mathrm{~mm}$ in diameter. In fact, energy sealing is generally applied in addition to suture ligation at the proximal side of cut vessels during chest surgery. Whether 1-step direct sealing without extra reinforcement is effective and safe for sealing pulmonary vessel branches, however, has not been supported by evidence.

We were particularly concerned about postoperative bleeding, and one of our patients bled from a pulmonary
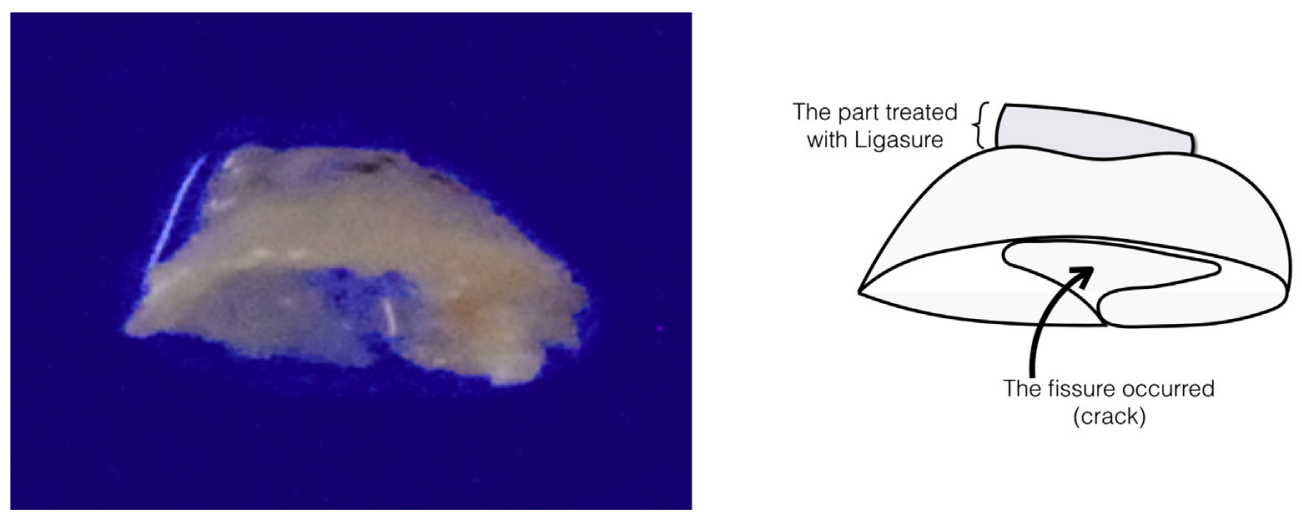

FIGURE 4. Macroscopic findings of pulmonary artery stump where postoperative bleeding was generated. 


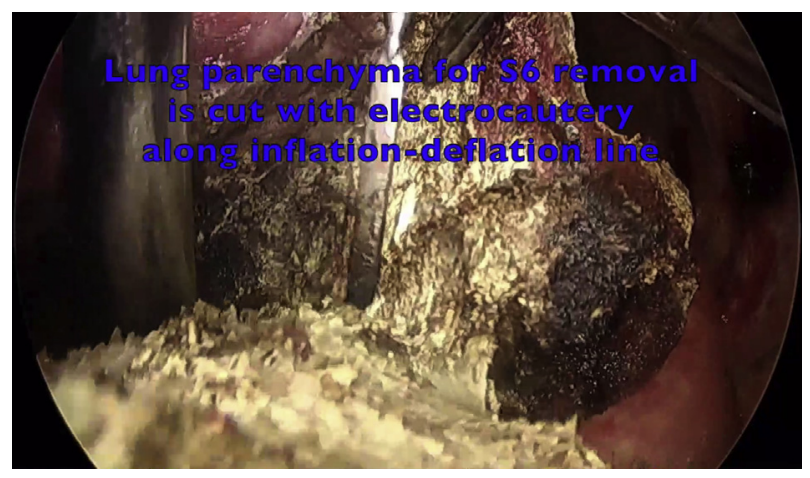

VIDEO 1. Left S6 (the superior segment of the lower lobe) segmentectomy for small-sized clinical stage IA non-small cell lung cancer using energy device to seal branches of the pulmonary arteries and veins. See the following article for details of the procedure: Okada M, Mimura T, Ikegaki J, Katoh H, Itoh $\mathrm{H}$, Tsubota $\mathrm{N}$. A novel video-assisted anatomic segmentectomy technique: selective segmental inflation via bronchofiberoptic jet followed by cautery cutting. J Thorac Cardiovasc Surg. 2007;133:753-8. Video available at: https://www.jtcvs.org/article/S0022-5223(18)31038-9/fulltext.

artery with a 6-mm diameter that was sealed with an energy device. This patient had systemic arteriosclerosis and a history of 2 brain hemorrhages, and high blood pressure was inadvertently provoked during recovery from anesthesia, which probably triggered the bleeding. Video playback and macroscopic investigation of the tissue notably confirmed that an area adjacent to the sealed zone was the source of the bleeding. Histopathologic findings of the tissue showed that the arterial wall in the area adjacent to the sealed zone where the bleeding occurred consisted of a thin layer of adventitia with no media or intima and was susceptible to hemorrhage. Such an area was not recognized on the pulmonary vein, which is worthy of note. Tsunezuka and coworkers found that burst pressure for pulmonary arteries was significantly higher in those with diameters of less than $5 \mathrm{~mm}$ than for those larger than $5 \mathrm{~mm}$ in a total of 203 vessels in resected lungs divided with a LigaSure device. ${ }^{10}$ Lesser and associates examined the safety of the LigaSure instrument for pulmonary artery sealing in 30 patients who underwent open lung lobectomy. ${ }^{11}$ They found a $50 \%$ lower burst pressure in sealed pulmonary arteries with diameters of 6 to $8 \mathrm{~mm}$ than in those 3 to $5 \mathrm{~mm}$. Toishi and colleagues $^{9}$ reported a significantly higher burst pressure in LigaSure-sealed stumps that were smaller than $5 \mathrm{~mm}$ than in those at least $5 \mathrm{~mm}$ during open lobectomy. Because the risk of bleeding depended on vessel size, we lowered the maximal diameter of the target pulmonary arteries to $5 \mathrm{~mm}$ in the cohort 2 study. Schuchert and colleagues ${ }^{12,13}$ retrospectively studied the effects of energy devices in 211 anatomic lung resections and could not directly attribute any complications or deaths to energy sealing. Other studies have found similar outcomes. ${ }^{9-11}$ None of the patients in the prospective study reported here died of surgery-related causes, with no operative mortality (within 30 days after surgery), and no serious complications arose in cohort 2 .

This study has some limitations. To implement such a study with only a single surgeon would be unrealistic, and the inclusion of several surgeons implies differences in surgical procedures, surgical proficiency of the operators, and the method of using the energy sealing device. These factors have more meaning through the standardization. The status of vessels may have varied before being measured, and the size of pulmonary vessels was measured by the same individual, which could create bias. The intraoperative procedure should be implemented carefully, because bleeding could occur as a result of contact between the stump and other instruments.

In conclusion, pulmonary arteries and veins that are as large as 5 and $7 \mathrm{~mm}$ wide, respectively, can be safely separated and sealed with energy devices. Energy sealing can potentially confer simpler vascular treatment, which could reduce intraoperative stress for surgeons.

\section{Webcast}

You can watch a Webcast of this AATS meeting presentation by going to: https://aats.blob.core.windows.net/media/18 Apr30/25ABC\%202.General\%20Thoracic\%20SS/S69\% 20-\%20Part\%201/S69_4_webcast_025828707.mp4.

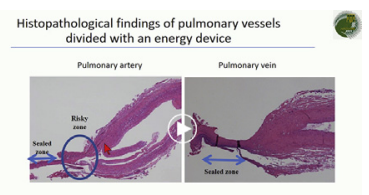

\section{Conflict of Interest Statement}

Drs Okada and Suzuki have received grants from Medtronic-Covidien, Inc, for consulting or advisory role and research funding. All other authors have nothing to disclose with regard to commercial support.

\section{References}

1. Okada M, Koike T, Higashiyama M, Yamato Y, Kodama K, Tsubota N. Radical sublobar resection for small-sized non-small cell lung cancer: a multicenter study. J Thorac Cardiovasc Surg. 2006;132:769-75.

2. Okada M, Mimura T, Ikegaki J, Katoh H, Itoh H, Tsubota N. A novel videoassisted anatomic segmentectomy technique: selective segmental inflation via bronchofiberoptic jet followed by cautery cutting. J Thorac Cardiovasc Surg. 2007; 133:753-8

3. Goudie E, Thiffault V, Jouquan A, Lafontaine E, Ferraro P, Liberman M. Pulmonary artery sealing with ultrasonic energy in open lobectomy: a phase I clinical trial. J Thorac Cardiovasc Surg. 2017;153:1600-7.

4. Bertolaccini L, Viti A, Cavallo A, Terzi A. Results of Li-Tho trial: a prospective randomized study on effectiveness of LigaSure ${ }^{\circledR}$ in lung resections. Eur J Car diothorac Surg. 2014;45:693-8; discussion 698.

5. Liberman M, Khereba M, Goudie E, Kazakov J, Thiffault V, Lafontaine E, et al. Pilot study of pulmonary arterial branch sealing using energy devices in an ex vivo model. J Thorac Cardiovasc Surg. 2014;148:3219-23. 
6. Cassivi SD, Pierson KE, Lechtenberg BJ, Nichols FC III, Shen KR, Allen MS, et al. Safety of air travel in the immediate postoperative period after anatomic pulmonary resection. J Thorac Cardiovasc Surg. 2017;153:1191-6.e1.

7. Tsutani Y, Mimura T, Kai Y, Ito M, Misumi K, Miyata Y, et al. Outcomes after lobar versus sublobar resection for clinical stage I non-small cell lung cancer in patients with interstitial lung disease. J Thorac Cardiovasc Surg. 2017;154: 1089-96.e1.

8. Committee for Scientific Affairs, Ueda Y, Fujii Y, Kuwano H. Thoracic and cardiovascular surgery in Japan during 2007. Annual report by the Japanese Association for Thoracic Surgery. Gen Thorac Cardiovasc Surg. 2009;57: 488-513. Erratum in: Gen Thorac Cardiovasc Surg. 2016;64:763-6.

9. Toishi M, Yoshida K, Agatsuma H, Sakaizawa T, Eguchi T, Saito G, et al. Usefulness of vessel-sealing devices for $\leq 7 \mathrm{~mm}$ diameter vessels: a randomized controlled trial for human thoracoscopic lobectomy in primary lung cancer. Interact Cardiovasc Thorac Surg. 2014;19:448-55.

10. Tsunezuka Y, Waseda R, Yachi T. Electrothermal bipolar vessel sealing device LigaSureV for pulmonary artery ligation-burst pressure and clinical experiences in complete video-assisted thoracoscopic major lung resection for lung cancer. Interact Cardiovasc Thorac Surg. 2010;11:229-33.

11. Lesser TG, Wolfram F, Boltze C. Sealing of pulmonary arteries with LigaSure: in vivo and ex vivo examinations. J Thorac Cardiovasc Surg. 2013;145:1525-8.

12. Schuchert MJ, Abbas G, Pettiford BL, Luketich JD, Landreneau RJ. Preliminary results of anatomic lung resection using energy-based tissue and vessel coagulative fusion technology. J Thorac Cardiovasc Surg. 2010;140:1168-73.

13. Schuchert MJ, Abbas G, Landreneau JP, Luketich JD, Landreneau RJ. Use of energy-based coagulative fusion technology and lung sealants during anatomic lung resection. J Thorac Cardiovasc Surg. 2012;144:S48-51.

Key Words: lung cancer, segmentectomy, energy device, vascular sealing

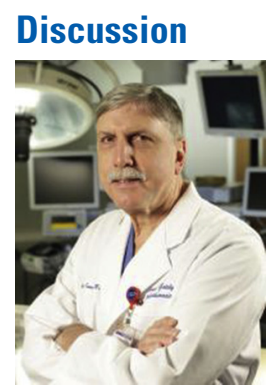

Dr Scott J. Swanson (Boston, Mass). Thank you for the opportunity to discuss the article. It was very well done. I have no conflicts. I have a comment and 2 questions.

The use of energy to divide pulmonary vessels has been described for more 10 years. We published our work in an animal model 11 years ago, demonstrating safety out to 6 weeks with ultrasonic energy and followed this up in our published report 2 years ago of our human experience with only 1 intraoperative bleeding event where there was immediate separation, and we put a clip on it. There was no consequence, the patients did fine, and that was in 118 consecutive vessels. Since that report, we have accumulated experience in more than 500 patients without any issues. Thus the use of ultrasonic energy to divide pulmonary artery and vein branches smaller than $7 \mathrm{~mm}$ is safe, and it does help make video-assisted thoracoscopic lobectomy and segmentectomy easier and more likely to be adopted.

Dr Miyata and his group just reported their experience with a different form of energy, electrothermal, with a LigaSure device, which was first reported by Dr Landreneau several years ago. Dr Miyata and his group observed 1 problem, which they immediately took care of, and there was no problem after that. They adjusted the protocol and had no further problems.
My first question is this. With the ultrasonic shears, there is some technical learning that goes on to make it easy to do; there are tricks and tips you can learn, a learning curve. Do you find there is a learning curve for how to use the LigaSure device to divide pulmonary artery and pulmonary vein?

Dr Yoshihiro Miyata (Hiroshima, Japan). Thank you very much for the question. I have never used an ultrasonic device, but for me it is almost the same and very easy for us to do it with a LigaSure device. It is bipolar, but still the time is very short. My concern for the ultrasonic device is a cavitation issue. So I prefer a bipolar device to an ultrasonic device.

Dr Swanson. The second question has to do with the economics of this. Do you use the LigaSure device for any other parts of your operation? Do you harvest lymph nodes with it, do you divide small amounts of lung parenchyma with it, or do you only use it to divide small vessels?

Dr Miyata. Of course, this is a study, so we sometimes use the device just for the vessels, but normally in clinical practice we use a LigaSure device for lymph node dissection. So it is not just for arterial ligation and dissection.

Dr Swanson. Well, this is a very nice study, and it will continue to help us achieve more widespread adoption of minimally invasive lobectomy and segmentectomy, which as we will hear tomorrow in the CALGB [Cancer and Leukemia Group B] study approaches $80 \%$ in the United States now. So I am very appreciative of this work. It is going to help us do a better job. Thank you.

Dr Miyata. Thank you.

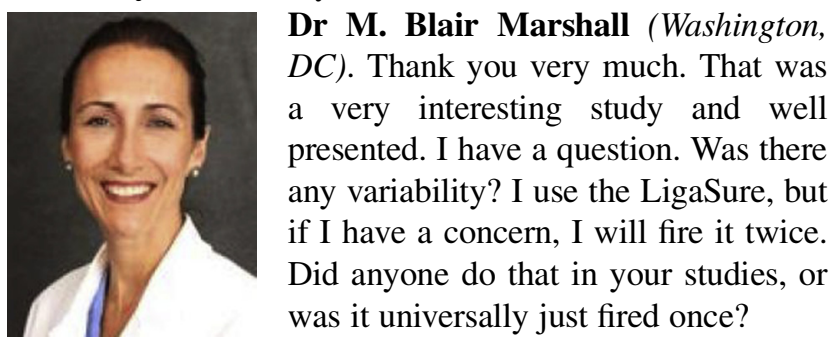

Dr Miyata. Excuse me, I didn't hear you.

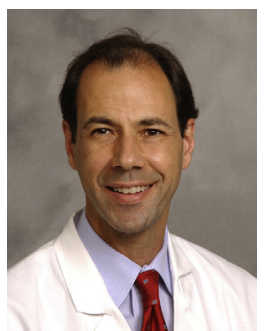

Dr D'Amico. Do you ever fire the LigaSure twice, hold it down, burn it, open it, hold it down, burn it and then cut it?

Dr Miyata. No. I tried it in an animal case as you said, twice burned it, but it is not effective enough and it is harmful a little bit, because the energy device produces heat and heat split leads to $0.6 \mathrm{~mm}$. So if we divide twice, the distal part would collapse out, maybe.

Dr Trevor Williams (Sacramento, Calif). I use the LigaSure routinely for my video-assisted thoracoscopic lobectomies, and I use the Harmonic on paraesophageal, just training stuff. But the LigaSure has this tendency to have tissue stick to it once you open the device, and with the pulmonary artery I can imagine that would cause me a 
lot of stress, and I am wondering how you dealt with that, or if it was an issue.

Dr Miyata. Yes, that is true, the LigaSure has some sticky coagulation in the jaw pattern. The Maryland jaw has an improvement compared to the blunt tip one. Of course, when we apply the LigaSure jaw to the vessels, especially the pulmonary artery, we ask the nurse to clean up the teeth before.

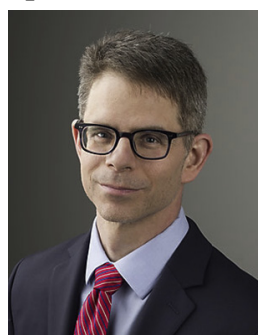

Dr Michael Stephen Kent (Boston, Mass). I just had a question about the precision of the measurements in your study. I am not sure that I could tell by eye the difference between a vessel that is $5 \mathrm{~mm}$ and one that is $6 \mathrm{~mm}$. So is there some objective way that you can document the size of the vessel, in your study or in real life, or is that just subjectively derived from the surgeon's experience? Thank you.

Dr Miyata. In this study we put the measure on the distal vessels, but in practice, the tip of the LigaSure jaw is $5 \mathrm{~mm}$ in width. So maybe compared to the tip of the LigaSure jaw we can measure the size of the vessels.

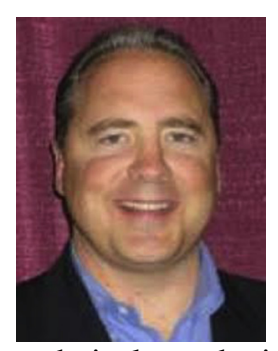

Dr Daniel L. Miller (Marietta, Ga). In this study, did you use the new LigaSure Nano? I know for the last 6 to 9 months, it has the new tip that does not have as much eschar buildup and so forth, and we have seen a big difference in coming off the pulmonary arteries. Also from a technical standpoint, we do not put tension on that vessel when we divide it, we try to relax on it a little bit, because sometimes you can see that ecchymosis or that little bit of a dissection in the adventitia. So I think it is very important when you are dividing these so you do not get into a late bleed not to do a double fire on it and just do a single fire. But the Nano has worked out very well. Do you have any experience with the new LigaSure tip?

Dr Miyata. I have never used that one, and I do not have any information about that.

Dr Miller. You do not have to clean it or anything like that.

Dr Miyata. Okay, thank you. I am going to try it. 